\title{
A Bayesian Approach to Service Selection for Secondary Users in Cognitive Radio Networks
}

\author{
Elaheh Homayounvala \\ Assistant Professor \\ Cyberspace Research Institute \\ Shahid Beheshti University \\ Tehran, Iran
}

\begin{abstract}
In cognitive radio networks where secondary users (SUs) use the time-frequency gaps of primary users' (PUs) licensed spectrum opportunistically, the experienced throughput of SUs depend not only on the traffic load of the PUs but also on the PUs' service type. Each service has its own pattern of channel usage, and if the SUs know the dominant pattern of primary channel usage, then they can make a better decision on choosing which service is better to be used at a specific time to get the best advantage of the primary channel, in terms of higher achievable throughput. However, it is difficult to inform directly SUs of PUs' dominant used services in each area, for practical reasons. This paper proposes a learning mechanism embedded in SUs to sense the primary channel for a specific length of time. This algorithm recommends the SUs upon sensing a free primary channel, to choose the best service in order to get the best performance, in terms of maximum achieved throughput and the minimum experienced delay. The proposed learning mechanism is based on a Bayesian approach that can predict the performance of a requested service for a given SU. Simulation results show that this service selection method outperforms the blind opportunistic SU service selection, significantly.
\end{abstract}

Keywords-Cognitive Radio; Service Selection; Bayesian Networks

\section{INTRODUCTION}

Efficient utilization of the scarce frequency spectrum resources has been the main reason to attract many researchers' interest to study dynamic spectrum access methods [1, 2]. Measurements have shown that the licensed parts of the spectrum in which conventional spectrum allocation methods are used by operators, are under-utilized $[3,4]$. Cognitive radio systems then are introduced to use the available spectrum temporarily not in use by licensed users, through intelligent channel sensing. In these systems, secondary users (SUs) are allowed to utilize the spectrum, as far as their transmissions do not have any harmful impact on the primary users' (PUs) operation $[5,6]$. Therefore, the effective detection of the PU's spectrum opportunities (holes), also called channel sensing or opportunity discovery, has a critical role in efficient usage of the valuable under-utilized parts of the spectrum and there are many research works focused on this topic [7].

The accurate sensing of the present status of the licensed channel is not the only challenge in cognitive radio networks (CRN). Assuming that the spectrum usage granularity of SUs is infinitive, then the effective throughput of SUs in a CRN depends only on the total duration of unused parts of the channel, which itself depends on the PUs traffic load. However, in practice, there is a minimum free channel time interval which is needed for different services of SUs to operate properly. This minimum duration depends on the structure of the secondary network as well as the service types and QoS requirements of SU services. In this case, the effective throughput of SUs also depends on the distribution of free parts of the licensed channel (Fig. 1). Ideally, in order to increase the achieved throughput of SUs, it is better to decrease this minimum required time interval by choosing proper physical layer parameters and utilizing less delay sensitive services. In practice, however, there is no control neither on secondary network structure, nor on its service requirements. Secondary network is a network just like the primary one, with the only difference that SUs do not have a licensed spectrum to work on. Therefore, if SUs have some knowledge about the distribution of the free channel time intervals, then they can make a better decision on choosing the right service to be used in a specific time. It is difficult to directly inform SUs of PUs' dominant used services and statistics of free channel time intervals in each area, for practical reasons.

This paper proposes a learning mechanism embedded in SUs to sense the primary channel for a specific length of time. Then, the algorithm recommends the SUs upon sensing a free primary channel, to choose the best service in order to get the best performance, in terms of achieved throughput. The proposed learning mechanism is based on a Bayesian approach that can predict the performance of a requested service for a given SU.

The rest of this paper is organized as follows. In section II, Bayesian networks and their application in CRN are briefly reviewed. Section III describes the system model as well as the proposed method to help SUs make a decision on choosing the best service at each specific time. Section IV represents the simulation results of the proposed service selection algorithm and finally, the paper is concluded in section $\mathrm{V}$. 


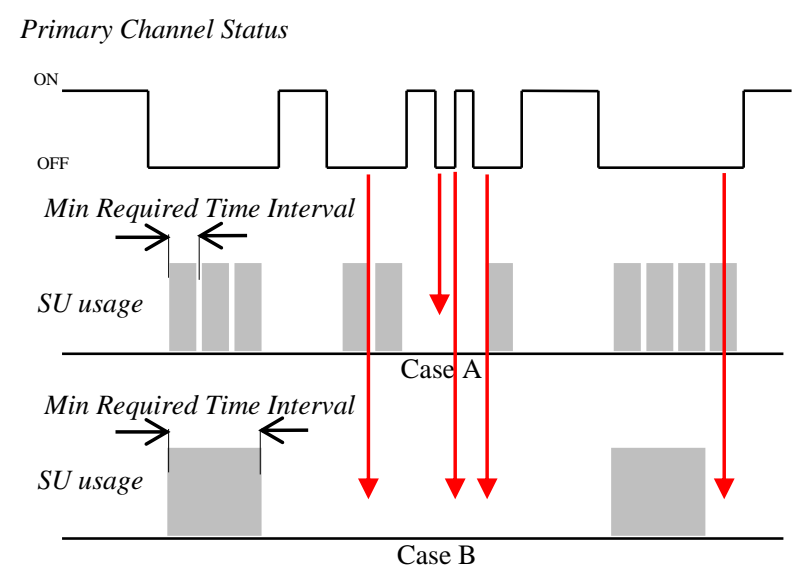

Fig. 1. Comparison of SU spectrum usage for different min required time interval values. Arrows show the "opportunities" of primary channel being off, but SUs cannot use the channel because of their min required time intervals

\section{BAYESIAN NETWORKS}

Bayesian networks can deal with two problems of uncertainty and complexity. "The Bayesian network formalism was invented to allow efficient representation of, and rigorous reasoning with, uncertain knowledge" [8]. Bayesian networks are applied in many different applications and domains such as diagnosis, forecasting, automated vision, sensor fusion, manufacturing control and also mobile communications. Efficient monitoring, control and management of telecommunication networks [9], and network fault diagnosis, analysis and predictions $[10,11]$ are two main categories of applications of Bayesian network formalism in mobile communications. In addition to the above applications, we introduced a new application of Bayesian networks for modeling user preferences for radio access selection $[12,13]$ which was later extended [14, 15]. Bayesian networks have been applied in CRNs in localization [16], channel estimation [17], spectrum sensing [18] and channel selection [19] to name a few.

A Bayesian Network consists of two parts. The first part is the graphical representation, which is a directed acyclic graph. This is the qualitative part of the network. Secondly, conditional probability functions of each node in the graph, which form the quantitative part of a Bayesian network. In a Bayesian network, when node A is connected to node $\mathrm{B}$ with a directed arc from $\mathrm{A}$ to $\mathrm{B}$, it usually means that node $\mathrm{A}$ causes node B. Node A is called the parent node and node B is called the child node. Conditional probability tables, in case of a discrete model, and conditional probability distributions in case of a continuous model, specify the probability of each child node conditioned on all possible combinations of values for all of its parent nodes.

The probabilities encoded by a Bayesian network can be learned from data. New information is combined with all previously known information using the Bayes' theorem [20]. Thomas Bayes, an 18th century British mathematician, presented Bayes' theorem, which is mathematically expressed as follows:

$$
P(H \mid E, C)=\frac{P(H \mid C) P(E \mid H, C)}{P(E \mid C)}
$$

Applying the Bayes' theorem we can update our belief in hypothesis $\mathrm{H}$ given an additional evidence $\mathrm{E}$ and the background context $\mathrm{C}$. $\mathrm{P}(\mathrm{H} \mid \mathrm{E}, \mathrm{C})$ represents the posterior probability of the hypothesis given the evidence. $\mathrm{P}(\mathrm{E} \mid \mathrm{H})$ is the likelihood of the evidence given the hypothesis. $\mathrm{P}(\mathrm{H})$ represents the prior probability of the hypothesis and $\mathrm{P}(\mathrm{E})$ is the normalizing constant. Probabilistic inference is the most common task performed by the aid of Bayesian networks. Inference in Bayesian networks answers the questions of the probability of a variable based on given observations of other variables.

\section{SYSTEM MODEL}

In this section the Bayesian network applied for proposed service selection algorithm for SUs in a CRN is explained. Bayesian networks have the ability to address problems of uncertainty. The uncertainty in this problem domain comes from two aspects. First there is uncertainty in service type used by PUs or requested by SUs. Secondly, call duration time and call arrivals for PUs are random. Therefore, the Bayesian view of the probability, that interprets probabilities as the "degree of belief" about events in the world, seems suitable for SUs' service selection. In such model, data is used to strengthen or weaken these degrees of belief.

The Bayesian network designed for this problem is illustrated in Fig 2. Without loss of generality, this paper defines the metric: channel utilization (CU) for each service in the secondary network, as the ratio of the time that channel is used for that service, to the time that channel is free for secondary usage. Therefore, this metric is independent of the primary traffic load and shows how efficient a secondary network with a specific service can use the primary spectrum holes. As it can be seen in this model, the CU of the channel depends on two parameters of PU service ratio (PUSR) and SU service type (SUST). PUSR ${ }_{\mathrm{i}}$ is the ratio of the time that primary channel is occupied to service $_{i}$ to the whole observation window. SUST represents the type of services that SUs can request.

Hence, the Bayesian inference in this model can answer the questions of the probability of the channel utilization being more than a given threshold for given service type $S j$ and a given PUSR. PUSR must be measured by sensing the channel for a specific window frame. Then, we refer to conditional probability table of $\mathrm{CU}$ node to see the value of the following term, for a given threshold:

$$
\text { Predicted_CU } U_{\text {th }, j}=P\left(C U \mid P U S R=p_{i}, S U S T=s_{j}\right)
$$

This is the probability of $\mathrm{CU}$, predicted by our model to be used for the $j^{\text {th }}$ service selection. In particular, if the value of this Predicted_CU th.i $_{i}$ is more than a threshold, then $S_{i}$ is recommended to be used in secondary network, otherwise $S_{i}$ is not recommended, which means that in this case, in order to have a CU more than the given threshold, this is not recommended to use $S_{i}$ in secondary network. This test can be checked for different SUTS values, when there are more than two services in the secondary network. 


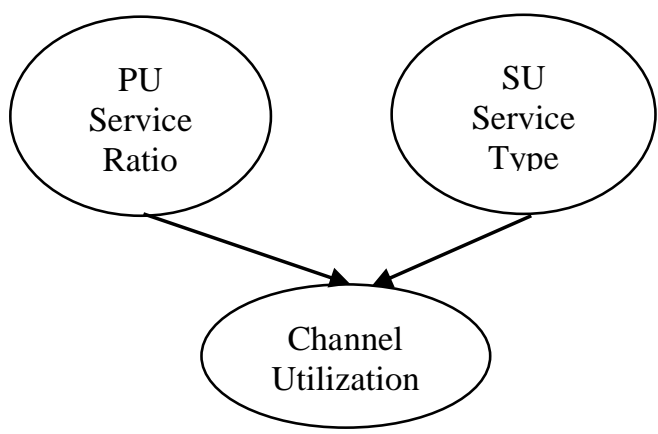

Fig. 2. Bayesian network for SUs' service selection in CRN

The smaller "mean call duration time" in the $j^{\text {th }}$ service, and the more delay resistant the $j^{\text {th }}$ service, the larger Predicted_CU $\mathrm{Cthi}_{i .}$ Generally speaking, in secondary networks, using delay tolerant services by short mean call durations has the lowest risk of blocking and yields to the largest channel utilization. However, when there are different types of services with different QoS requirements queued at SUs, it is important to choose the best service at each time in order to reach the largest throughput and the smallest delay for services. Therefore, when there are more than two services in the secondary network, service selection does not depend on Predicted_CU ${ }_{t h, j}$ only, but also on the $j^{\text {th }}$ queue length as well.

\section{Simulation Results}

In this section the simulation setup and its parameters are explained. A primary channel with the states of " 1 " and " 0 " is assumed to show the "busy" and "free" status, respectively. It is also assumed that the primary channel is used by PUs with two types of services; service 1 whose call arrival times follow a Poisson distribution with $\lambda_{1}=10$ calls $/ \mathrm{min}$ with exponentially distributed call duration times with a mean of $\mathrm{T}_{\text {lave }}=\Delta t \mathrm{sec}$, and service 2 with $\lambda_{2}=0.5$ and $\mathrm{T}_{2 a v e}=50 \Delta t$, where $\Delta t$ is the time resolution of our time driven simulation. Service1 represents a typical short term service with a frequent call arrival, such as voice and some interactive gaming. Notice that the important point here is the traffic statistics rather than the QoS requirements of the service, as in a CRN, the PUs' services always have the priority over SUs ones, even though they are not delay sensitive, inherently. Service2 represents a typical long term service whose packets are transferred in bulks, such as file transfer or video downloading. These services take a more considerable continuous time interval of the channel, independent of if they are delay tolerable or not. Then, we have modeled a time window in which the channel is occupied by these two services, service1 and service2, for a given probability. Figure 3 shows primary channel status for a typical 50\% mixed traffic case of these two services.

For secondary network, we assume the same two services as the primary network uses, for the sake of simplicity. We also set the min required time intervals of servicel and service 2 to $\mathrm{T}_{1 \text { min }}=\mathrm{T}_{1 \text { ave }}$ and $\mathrm{T}_{2 \min }=\mathrm{T}_{2 \text { ave }}$, respectively. $\mathrm{We}$ assume that SUs always have data packets of service1 and/or service 2 in queue, ready to use the free primary channel. Table 1 shows the SU channel utilization (CU) for service2 for different combinations of service 1 and service 2 traffics in the primary channel.
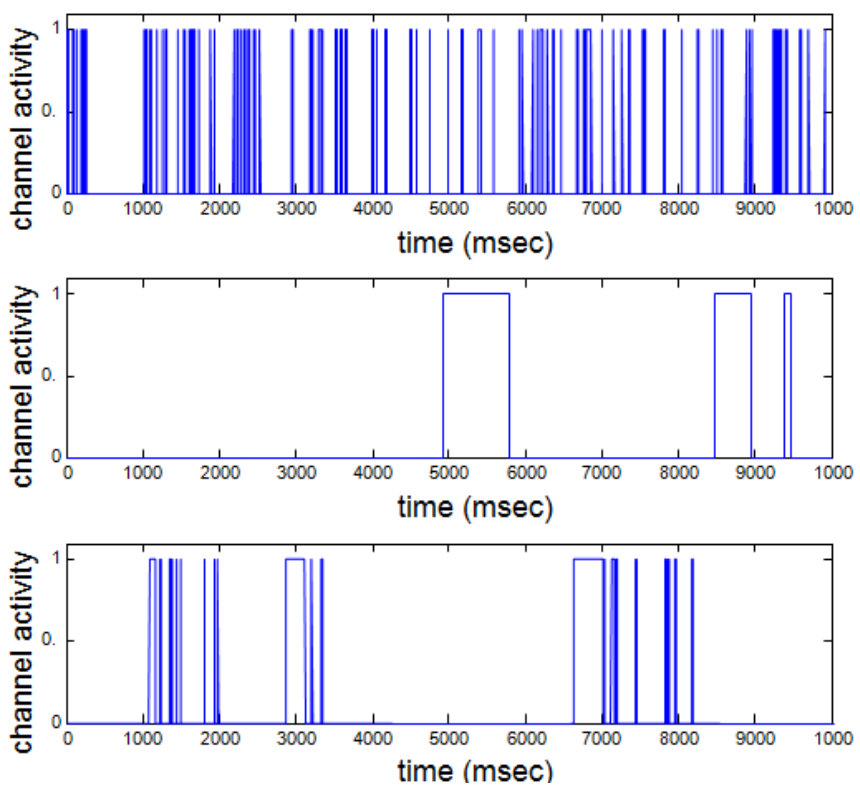

Fig. 3. A typical primary channel status: a) $100 \%$ service1 b) $100 \%$ service 2 c) $50 \%$ mixed scenario

network. For service1, CU is 0.98 in all PUSR values. It can be seen from these conditional probability table that channel utilization for service1 SUs is always high, independent of service combination in the primary network. This is because $\mathrm{T}_{1 \min } \ll \mathrm{T}_{2 \min }$ in our simulation, which means that service 1 can use most of the primary channel opportunities while service2 loses some of these opportunities because of its longer min required time interval. This loss increases as the ratio of service 2 in primary channel traffic goes up.

In the last part of the simulation, two CRNs are compared. The first one uses a blind decision making about SUs services and the second CRN chooses the SUs service based on the Bayesian learning algorithm whose parameters are tuned according to the results of an observation time such as what have been shown in Fig. 3. The simulation results for an observation window, which contains at least 1000 channel status changes, show that the utilization of the proposed service selection algorithm outperforms the random service selection method for at least $20 \%$.

TABLE I. TYPICAL VALUES OF CHANNEL UtILIZATION (CU) USING SERVICE2 FOR DIFFERENT COMBINATIONS OF SERVICE1 AND SERVICE2 TRAFFICS IN THE PRIMARY NETWORK. ITR\#I IS THE ITH ITERATION

\begin{tabular}{|l|l|l|l|l|l|l|l|l|l|}
\hline $\begin{array}{l}\text { PUS } \\
\text { R }\end{array}$ & $\begin{array}{l}\text { itr\# } \\
1\end{array}$ & $\begin{array}{l}\text { itr\# } \\
2\end{array}$ & $\begin{array}{l}\text { itr\# } \\
3\end{array}$ & $\begin{array}{l}\text { itr\# } \\
4\end{array}$ & $\begin{array}{l}\text { itr\# } \\
5\end{array}$ & $\begin{array}{l}\text { itr\# } \\
6\end{array}$ & $\begin{array}{l}\text { itr\# } \\
7\end{array}$ & $\ldots$ & $\begin{array}{l}\text { itr\#10 } \\
0\end{array}$ \\
\hline $80 \%$ & .82 & .83 & .79 & .87 & .86 & .76 & .84 & & .75 \\
\hline $85 \%$ & .75 & .84 & .79 & .81 & .77 & .73 & .74 & & .80 \\
\hline $90 \%$ & .51 & .65 & .73 & .56 & .71 & .83 & .64 & & .71 \\
\hline $92 \%$ & .73 & .67 & .73 & .72 & .65 & .63 & .65 & & .88 \\
\hline $95 \%$ & .43 & .51 & .36 & .29 & .82 & .54 & .44 & & .43 \\
\hline $98 \%$ & .43 & .25 & .32 & .33 & .30 & .19 & .29 & & .28 \\
\hline $99 \%$ & .19 & .13 & .16 & .15 & .10 & .18 & .16 & & .25 \\
\hline $100 \%$ & .05 & .05 & .04 & .04 & .02 & .02 & .01 & & .04 \\
\hline
\end{tabular}




\section{CONCLUSION}

This paper utilized the statistics of the primary channel occupancy to choose the secondary services in a way that the channel utilization of secondary users in a cognitive radio network increases. We have used a Bayesian network to model the channel utilization based on different possible services of primary and secondary networks. The simulations are performed for a simple case of two services, but they can be easily extended in future work to the case of multiple services and users in the primary network, to enhance the performance of the system in terms of dropping probability and experienced delay, as well.

\section{REFERENCES}

[1] I. F. Akyildiz, W.-Y. Lee, M. C. Vuran, and S. Mohantly, "Next generation/ dynamic spectrum access/cognitive radio wireless network:a survey," Elsevier Computer Networks, vol. 50, pp. 2127-2159, Sept. 2006.

[2] A. Attar, M. S. A. Ghorashi, Sooriyabandara and A. H. Aghvami, "Challenges of real-time secondary usage of spectrum", Computer Networks, vol. 52, no. 4, pp. 816-830, 2008.

[3] M. McHenry, "Frequency agile spectrum access technologies," in Proc. FCC Workshop on Cognitive Radio, May 2003.

[4] M. A. McHenry, "NSF spectrum occupancy measurements project summary," Shared Spectrum Company technical report, Aug. 2005. Available at http://www.sharedspectrum.com/

[5] J. Mitola, "Cognitive radio for flexible mobile multimedia communications, " in Proc. IEEE Int. Workshop Mobile Multimedia Communications, pp. 3-10, 1999.

[6] J. Mitola, "Cognitive radio: an integerated agent architecture for software defined radio," Doctor of Technology, Royal Inst. Technol. (KTH), Stockholm, Sweden, 2000.

[7] T. Yucek and H. Arslan, "A survey of spectrum sensing algorithms for cognitive radio applications, "IEEE Communications Surveys and Tutorials, vol. 11, no. 1, pp. 116-130, First Quarter 2009.

[8] S. Russell and P. Norvig, Artificial Intelligence a Modern Approach, Prentice Hall series in Artificial Intelligence, 2003.

[9] A. Bashar, GP. Parr,S.I McClean, B.W. Scotney, D. Nauck, "Knowledge discovery using Bayesian network framework for intelligent telecommunication network management" in Proc. Of Springer LNCS LNAI series, $4^{\text {th }}$ Internaional Conference on Knowledge Science, Engineering anf Management (KSEM 2010), Belfast, UK, pp. 518-529, September 2010.

[10] R.M. Khanafer, B. Solana, B., J. Triola, R. Barco, L. Moltsen, Z. Altman, P. Lazaro, " Automated diagnosis for UMTS networks using Bayesian network approach", IEEE Trans. Vehicular Technology, vol. 57, no. 4, pp. $2451-2461,2008$.

[11] A. Sedano-Frade, J. González-Ordás, P. Arozarena-Llopis, S. GarcíaGómez and A. Carrera-Barroso, "Distributed Bayesian diagnosis for telecommunication networks", Advances in Practical Applications of Agents and Multiagent Systems, AISC, vol. 70, pp 231-240, 2010.

[12] E. Homayounvala, A.H. Aghvami, "User preference modelling for access selection in multiple radio access environments", IEICE Transactions on Communications, Special Section on Software Defined Radio Technology and Its Applications, vol. EE88-B, no. 11, Nov. 2005, pp. 4186-4193.

[13] E. Homayounvala, S. A. Ghorashi, A. H. Aghvami, "A Bayesian approach to modelling user preferences for reconfiguration", $E 2 R$ Workshop, Barcelona, Spain, September 2004.

[14] A. Koutsorodi, E. Adamopoulou, K. Demestichas, M. Theologou, "Service configuration and user profiling in 4G terminals". Wireless Personal Communications, Vol 43, No 4, p.p.1303-1321, Dec. 2007

[15] K. Demestichas, A. Koutsorodi, E. Adamopoulou, M. Theologou, "Modelling user preferences and configuring services in B3G devices", Wireless Networks, July 2007.

[16] S. Kandeepan, et. al. "Bayesian Tracking in Cooperative Localization for Cognitive Radio Networks, "IEEE $69^{\text {th }}$ Vehicular Technology Conference, VTC Spring Barcelona, pp. 1 - 5, 26-29 April 2009.

[17] P. Demestichas et. al, "Enhancing channel estimation in cognitive radio systems by means of Bayesian networks, " Journal of Wireless Personal Communications, vol. 49, no. 1, April 2009.

[18] Zhenghao Zhang Husheng Li Depeng Yang Changxing Pei, "Spacetime Bayesian compressed spectrum sensing for wideband cognitive radio networks, " IEEE Symposium of New Frontiers in Dynamic Spectrum, pp. 1 - 11, Singapore, 6-9 April 2010.

[19] Guangxiang Yuan et. al., "Multi-User cooperation for channel selection in cognitive radio networks: a Bayesian approach," IEEE Globecom 2010 .

[20] R. L. Winkler, "Introduction to Bayesian Inference and Decision", Series in Quantities Methods for Decision Making, Holt, Rinehart and Winston Inc, 1972. 